\title{
Reperfusion after pulmonary embolism - long-term follow-up, risk factors, clinical impact
}

\author{
Jan Mrozek ${ }^{\mathrm{a}, \mathrm{b}}$, Jana Petrova ${ }^{\mathrm{b}}$, Jana Vaclavkovac, Vladimir Janovskyc, Lubos Kraus ${ }^{\mathrm{d}}$, Pavel Jansa ${ }^{\mathrm{e}}$
}

Background and Aim. Thromboembolic disease is the third most common cardiovascular disorder and deep vein thrombosis carries the risk of pulmonary embolism (PE). Questions related to reperfusion after PE remain, especially risk factors. Incomplete reperfusion after PE is closely related to the development of chronic thromboembolic pulmonary hypertension. The aim of this study was to determine the relation between reperfusion after PE in the long term over a period of 24 months, laboratory results and clinical risk factors found during the initial PE event.

Patients and Methods. 85 consecutive patients with a first episode of acute PE, diagnosed at 4 cardiology clinics, were followed up using clinical evaluation, scintigraphy and echocardiography $(6,12$ and 24 months after the PE. 35 patients were in the low risk category (41\%), $42(49 \%)$ in the intermediate risk group and $8(9 \%)$ in the high risk category. Results. Perfusion defects persisted in 20 patients (26\%) after 6 months, in 19 patients (25\%) after 12 months and in 14 patients (19\%) after 24 months. The incidence was more frequent in older patients, with more serious (higher risk) $\mathrm{PE}$, increased right ventricular internal diameter during the initial episode, and more significant tricuspid insufficiency in the initial echocardiography. Notably, higher hemoglobin levels were also shown as a significant risk factor. The presence of perfusion defects after 24 months correlated with a concurrent higher pulmonary pressure but not with either patient function or adverse events (recurrence of $\mathrm{PE}$, re-hospitalization or bleeding). In 3 cases ( $4 \%$ of patients), long-term echocardiographic evidence of pulmonary hypertension was detected.

Conclusion. Even after 24 months from acute PE with adequate anticoagulation treatment, incomplete reperfusion was found in $19 \%$ of patients with a corresponding risk of chronic thromboembolic pulmonary disease and hypertension.

Key words: pulmonary embolism, incomplete reperfusion, risk factor

Received: October 24, 2017; Accepted: January 8, 2018; Available online: January 24, 2018

https://doi.org/10.5507/bp.2018.001

${ }^{a}$ Department of Cardiovascular Diseases, University Hospital Ostrava, Czech Republic

${ }^{b}$ Department of Cardiology, Municipal Hospital, Ostrava, Czech Republic

'Department of Internal Medicine, Silesian Hospital Opava, Czech Republic

${ }^{d}$ Department of Internal Medicine, Hospital Novy Jicin, Czech Republic

${ }^{e}$ Clinical Department of Cardiology and Angiology, $1^{\text {st }}$ Faculty of Medicine, $2^{\text {nd }}$ Medical Department, Charles University, Prague, Czech Republic

Corresponding author: Jan Mrozek, e-mail: honzamrozek@email.cz

\section{INTRODUCTION}

Thromboembolic disease (TED) is the third most common cardiovascular condition with an incidence of 100-200 cases per 100,000 inhabitants per year. It plays a significant role in cardiovascular morbidity and mortality, and considerable resources are being spent on its treatment and prevention.

In the first hours after the onset of the pulmonary embolism (PE), the cause of death is usually an obstructive shock with failure of the right ventricle. However, in the days following PE, most patients die of other illnesses (such as heart failure, malignancy, chronic obstructive pulmonary disease or infection) rather than of recurrence of $\mathrm{PE}\left(\right.$ ref. $\left.^{1}\right)$. The initial extent of pulmonary artery obstruction is not a predictor of long-term mortality ${ }^{1,2}$.

The only known long-term complication of PE is chronic thromboembolic pulmonary hypertension (CTEPH), which occurs in $2-3 \%$ of patients within two years of PE (ref.-6). The outlook in remaining PE patients is good, providing they receive appropriate treatment, and most of them (over $75 \%$ ) survive in the long-term without any impact on their condition or quality of life.

In most patients, effective anticoagulation and spontaneous fibrinolytic activity of the plasma leads to a gradual dissolution of the thrombi and to the complete recanalization of the pulmonary arteries.

The most commonly reported risk factors of incomplete reperfusion traditionally include the severity of the initial PE expressed as the obstruction index ${ }^{6-9}$, presence of central emboli and right ventricular enlargement on the initial CT $\operatorname{scan}^{10}$.

The aim of our multicentric study was prospective monitoring of the reperfusion of the pulmonary arteries after the first pulmonary embolism event and correlating the results of such monitoring to laboratory results taken at the time of the initial PE and to clinical risk factors. 


\section{PATIENTS AND METHODS}

85 patients hospitalized in the internal medicine or cardiology departments of four hospitals (Ostrava City Hospital, University Hospital Ostrava, Silesian Hospital Opava and Nový Jičín Hospital) due to a first event of acute pulmonary embolism from December 2011 to June 2013 were included in the study. Diagnosis of acute PE was determined by CT angiography and / or ventilationperfusion (VP) scintigraphy. The patients were fully informed and signed informed consent to the study procedures and further monitoring prior to enrollment in the study. The study protocol was approved by the ethics committee.

Pulmonary embolism was treated in the standard way according to recent ESC guidelines and recommended procedures. Upon release from the hospital, patients were to regularly attend cardiology clinics at the place of residence.

Follow-up examinations at the participating departments took place 6,12 , and 24 months after the initial $\mathrm{PE}$. The reperfusion, possible recurrence of $\mathrm{PE}$ and effectiveness of anticoagulation therapy in warfarin-treated patients (\% of INRs performed during outpatient checkups at the local clinic) were evaluated.

The baseline echocardiographic examination was performed during the initial hospitalization, follow-up echocardiographic examinations were performed after 6,12 , and 24 months. The pulmonary artery systolic pressure was estimated from tricuspid regurgitation flow velocity, with a correction for the estimated right atrial pressure.

The initial VP pulmonary scintigraphy was also performed during the initial hospitalization. Follow-up perfusion scintigraphies were carried out after 6, 12 and 24 months only where the previous scintigraphy revealed incomplete reperfusion or an interim PE recurrence. The presence of defects, their number and extent were recorded on the scans.

\section{Statistical analysis}

The statistical analysis was performed in IBM SPSS Statistics 24. Continuous variables were described using the known $\mathrm{N}$ and medians with the $5^{\text {th }}$ and $95^{\text {th }}$ percentiles, discrete category variables were described using absolute and relative frequency. Mean or median differences were recorded for ECHO, ECHO after 6, 12 and 24 months. The statistical significance of the differences was tested using the Wilcoxon paired test. The associations between variables measured during the initial examination and the incidence of perfusion defects at individual time points were tested using the Mann-Whitney $\mathrm{U}$ test and Fisher's exact test (for categorical variables) with significance level $\alpha=0.05$.

\section{RESULTS}

\section{Risk factors, initial treatment}

A total of 85 patients were prospectively included, of who $48(57 \%)$ were women. The median age was 60 years. The TED and CTEPH risk factors were represented as follows: positive family history of TED in 18 (21) patients, personal history of DVT in $9(10.6 \%)$ patients, thyreopathy in $6(7 \%)$ patients, and malignancy in $4(5 \%)$ patients. Other well-recognized risk factors for thromboembolism and CTEPH were found only rarely, presence of pacemaker in 1 patient, and myeloproliferative disease in 1 patient. No other risk factors were present. The PE was provoked in 26 patients $(31 \%)$.

In $35(41 \%)$ patients, the PE was evaluated as low risk, in $42(49 \%)$ as intermediate and in $8(9 \%)$ patients as high risk.

Systemic thrombolysis was carried out in 14 (17\%) patients. The remaining patients received only anticoagulative treatment during hospitalization. The entry characteristics of the patient group are summarized in Table 1.

\section{Chronic treatment}

After 6 months, 76 out of 79 patients (96\%) were still anticoagulated, which 57 patients were treated with warfarin, the rest with other anticoagulants. 55 out of 77 patients $(71 \%)$ were on anticoagulation treatment after 12 months, and the treatment was ongoing in $27 / 74$ patients (37\%) after 24 months from the PE episode. In warfarin patients, effective anticoagulation treatment (at least $75 \%$ INR values over the period $>2$ ) was recorded only for 29 out of 57 patients (51\%) after 6 months, 25 out of 44 patients (57\%) in the 6-12 months period and 16 out of 18 patients ( $89 \%$ ) in the $12-24$ months period.

\section{Long-term clinical monitoring}

Out of the 85 patients, 79 were re-examined after 6 months, 77 patients after 12 months, and 74 after

Table 1. Entry characteristics of patients.

\begin{tabular}{|c|c|c|}
\hline \multicolumn{3}{|l|}{ Demography } \\
\hline \multirow[t]{2}{*}{ Sex } & Male & $37(44 \%)$ \\
\hline & Female & $48(56 \%)$ \\
\hline Age (years) & $\mathrm{n}=85$ & $60.0(27.0 ; 78.0)$ \\
\hline BMI $\left(\mathrm{kg} / \mathrm{m}^{2}\right)$ & $\mathrm{n}=82$ & $29.6(22.3 ; 38.5)$ \\
\hline \multicolumn{3}{|c|}{ Personal history } \\
\hline \multicolumn{2}{|c|}{ FH VTE } & $18(21 \%)$ \\
\hline \multicolumn{2}{|l|}{ DVT } & $9(11 \%)$ \\
\hline \multicolumn{2}{|l|}{ Thyreopathy } & $6(7 \%)$ \\
\hline \multirow[t]{5}{*}{$\mathrm{PE}$} & High risk & $8(9 \%)$ \\
\hline & Intermediate risk & $42(49 \%)$ \\
\hline & Low risk & $35(41 \%)$ \\
\hline & Provoked & $26(31 \%)$ \\
\hline & Unprovoked & $59(69 \%)$ \\
\hline \multicolumn{3}{|c|}{ Initial treatment } \\
\hline \multicolumn{2}{|c|}{ Anticoagulation } & $71(84 \%)$ \\
\hline \multicolumn{2}{|c|}{ Systemic thrombolysis } & $14(16 \%)$ \\
\hline
\end{tabular}

Continuous variables are described using number of patients $(\mathrm{N})$, medians ( $5^{\text {th }}$ and 95 th percentile). Category variables are described using absolute (relative) frequency.

BMI - body mass index, FH VTE - Family history of venous thromboembolism, DVT - deep vein thrombosis, PE - pulmonary embolism , $\mathrm{BNP}$ - Brain natriuretic peptide, $\mathrm{Hb}$ - hemoglobin 
24 months. 5 patients died during the study period (one during the initial hospitalization, one 6 months later, and 3 between $12^{\text {th }}$ and $24^{\text {th }}$ month). Contact with one patient was lost. In 5 cases, only telephone contact with verbal verification of the most important parameters (TED recurrence, bleeding and rehospitalization) was possible.

During follow-up, PE recurred in 7 ( $8 \%$ ) patients, 5 of whom between months 12 and 24, after the anticoagulation treatment ceased. Nine patients $(11 \%)$ developed bleeding. 23 (27\%) of patients were rehospitalized during the follow-up period. During the follow-up, patients were generally in good condition - the median NYHA class was 1 at all follow-up time points. Only 6 patients after 6 months and 5 patients after 12 and 24 months were NYHA class 3 or 4 .

\section{Echocardiography and scintigraphy examinations}

Most echocardiographic parameters were abnormal at the initial examination. The median of the right ventricular internal diameter index (RVIDd) was 34(26; 47) $\mathrm{mm}$, tricuspid annular plane systolic excursion (TAPSE) $19(15 ; 30) \mathrm{mm}$, right atrial dimension (RAD) 44(29; $53)$, tricuspid regurgitation (TR) $2.0(1.0 ; 3.5)$, and the median of pulmonary artery systolic pressure (PASP) estimate $40(15 ; 65) \mathrm{mmHg}$. Echocardiographic examinations after 6 months revealed a significant improvement in most echocardiographic parameters - compared with the initial measurements, median RVIDd reduction was $2(-14 ; 6) \mathrm{mm}$, median TAPSE improvement was $6(-9,20)$ $\mathrm{mm}$, RAD reduction was $3.0(-16 ; 18) \mathrm{mm}$, the severity of tricuspid regurgitation was improved by 0.5 degrees $(-2.0 ; 1.0)$, and the median PASP reduction was by 7 ( -35 , 12) $\mathrm{mmHg}$. Subsequent examinations (12, 24 months) did not show any further significant improvements over the first follow-up after 6 months.

The follow-up scintigraphies revealed a complete reperfusion in most patients. Perfusion defects persisted in $20(26 \%)$ patients after 6 months, 19(25\%) after 12 months and 14(19\%) patients after 24 months. A complete reperfusion was therefore reached in $80.8 \%$ patients. Also, the total number of perfusion defects dwindled from an initial 204 to 47 after 6 months, 41 after 12 months and 36 after 24 months.

Signs of a probable pulmonary hypertension were detected in 3 patients (4\%) with perfusion defects persisting even after 24 months (see Table 2). It is very likely that these patients developed CTEPH, however verification using invasive methods (right sided cardiac catheterization, pulmonary angiography) was impossible as all 3 patients refused the procedures ( 2 for a poor health condition, 1 for absence of any symptoms). Permanent anticoagulation treatment was recommended.

A comparison of patients with defects persisting even after 24 months and patients with complete reperfusion revealed the following significant differences: patients with persisting defects were older (age difference), the initial episode of PE was more frequently intermediate or high risk in these patients, they had a higher initial level of hemoglobin and the initial echocardiography revealed larger right ventricular internal dimension and more significant tricuspid regurgitation. No other variables (such as clinical risk factors of thromboembolic disease, other echocardiographic or laboratory parameters, thrombophilia or way of treatment) were significantly different. Also, the group with persisting perfusion defects did not differ significantly in the NYHA-rated functional status or other clinical parameters such as recurrence of pulmonary embolism or rehospitalization.

The median PASP values after 24 months were higher in the patients with persisting defects. There was however no significant difference in other echocardiography parameters such as right compartment dimensions or the function of the right ventricle. (Table 3 )

\section{DISCUSSION}

Reperfusion after PE, its risk factors, causes and consequences of incomplete reperfusion are discussed in a number of studies. The reported rate of complete reperfusion 6-12 months after PE ranges from 33 to $81 \%$, absence of any reperfusion is found in 3-16\% (ref. ${ }^{6,7,11}$ ). The wide range of results may be due to methodological issues - different patient classification, study period or different diagnostic methods (CT AG or VP scintigraphy). Scintigraphy was shown to be more sensitive but less specific in diagnosing perfusion defects and, therefore, has a tendency to overestimate the number of patients with persisting perfusion defects compared to CT AG (ref. ${ }^{12,13}$ ). Perfusion scintigraphy is more focused on perfusion on the microcirculation level, being able to capture more sensitively the obliteration of small arterioles that cannot be detected using CT AG; on the other hand, the pulmonary microcirculation is dependent on multiple factors including e.g. regional hypoxia resulting from damage to the pulmonary parenchyma. In this study group, perfusion

Table 2. Characteristics of CTEPH patients.

\begin{tabular}{|c|c|c|c|c|c|c|c|c|c|}
\hline Patient & Sex & $\begin{array}{c}\text { Age } \\
\text { (years) }\end{array}$ & $\begin{array}{c}\text { DVT } \\
\text { history }\end{array}$ & PE risk class & $\begin{array}{l}\text { Initial PE } \\
\text { treatment }\end{array}$ & $\begin{array}{l}\text { No. of initial } \\
\text { perfusion } \\
\text { defects }\end{array}$ & $\begin{array}{c}\text { No. of } \\
\text { perfusion } \\
\text { defects after } \\
24 \text { months }\end{array}$ & $\begin{array}{c}\text { Initial PASP } \\
\text { estimate } \\
(\mathrm{mmHg})\end{array}$ & $\begin{array}{l}\text { PASP esti- } \\
\text { mate after } \\
24 \text { months } \\
(\mathrm{mmHg})\end{array}$ \\
\hline 1 & Female & 69 & Yes & Intermediate & Anticoagulation & 4 & 4 & 42 & 55 \\
\hline 2 & Male & 36 & Yes & Intermediate & Anticoagulation & 5 & 5 & 80 & 45 \\
\hline 3 & Female & 80 & No & High & Thrombolysis & 2 & 2 & 62 & 47 \\
\hline
\end{tabular}


Table 3. Comparison of patients with and without persisting perfusion defects after 24 months.

\begin{tabular}{|c|c|c|c|c|c|}
\hline & & No $(n=59)$ & & Yes $(n=14)$ & $P$ \\
\hline \multicolumn{6}{|l|}{ Demography } \\
\hline Age & $\mathrm{n}=59$ & $55.0(27.0 ; 78.0)$ & $\mathrm{n}=14$ & $67.5(21.0 ; 78.0)$ & 0.020 \\
\hline Females & & $33(56 \%)$ & & $7(50 \%)$ & 0.770 \\
\hline BMI & $\mathrm{n}=58$ & $29.2(20.8 ; 38.5)$ & $\mathrm{n}=13$ & $29.8(24.6 ; 39.4)$ & 0.323 \\
\hline History of DVT & & $5(8 \%)$ & & $3(21 \%)$ & 0.175 \\
\hline PE risk High & & $4(7 \%)$ & & $2(14 \%)$ & \\
\hline Intermediate & & $24(41 \%)$ & & $10(71 \%)$ & 0.026 \\
\hline Low & & $31(53 \%)$ & & $2(14 \%)$ & \\
\hline Unprovoked PE & & $39(66 \%)$ & & $12(86 \%)$ & 0.204 \\
\hline \multicolumn{6}{|l|}{ Entry lab results } \\
\hline Troponin (ng/mL) & $\mathrm{n}=57$ & $0.0(0.0 ; 1.0)$ & $\mathrm{n}=12$ & $0.1(0.0 ; 2.3)$ & 0.737 \\
\hline $\mathrm{Hb}(\mathrm{g} / \mathrm{L})$ & $\mathrm{n}=59$ & $136.0(118.0 ; 159.0)$ & $\mathrm{n}=14$ & $144.5(123.0 ; 172.0)$ & 0.031 \\
\hline Thrombocytes $\left(1 * 10^{-9}\right)$ & $\mathrm{n}=59$ & $206.0(115.0 ; 370.0)$ & $\mathrm{n}=14$ & $217.5(130.0 ; 367.0)$ & 0.763 \\
\hline D-dimers $(\mu \mathrm{g} / \mathrm{L})$ & $\mathrm{n}=56$ & $1742.0(4.1 ; 7894.0)$ & $\mathrm{n}=8$ & $2229.5(1.0 ; 8$ 840.0) & 0.887 \\
\hline \multicolumn{6}{|l|}{ Initial ECHO } \\
\hline RVIDd (mm) & $\mathrm{n}=58$ & $33.5(23.0 ; 45.0)$ & $\mathrm{n}=14$ & $36.5(27.0 ; 54.0)$ & 0.044 \\
\hline TAPSE (mm) & $\mathrm{n}=44$ & $19.0(15.0 ; 30.0)$ & $\mathrm{n}=9$ & $17.0(13.0 ; 33.0)$ & 0.347 \\
\hline $\mathrm{RAD}(\mathrm{mm})$ & $\mathrm{n}=9$ & $40.0(32.0 ; 48.0)$ & $\mathrm{n}=33$ & $45.0(28.0 ; 58.0)$ & 0.110 \\
\hline TR (degree) & $\mathrm{n}=54$ & $1.5(1.0 ; 3.0)$ & $\mathrm{n}=13$ & $2.0(1.0 ; 27.0)$ & 0.018 \\
\hline PASP estimate (mmHg) & $\mathrm{n}=44$ & $35.5(15.0 ; 57.0)$ & $\mathrm{n}=13$ & $45.0(15.0 ; 80.0)$ & 0.170 \\
\hline \multicolumn{6}{|l|}{ Initial treatment } \\
\hline Systemic thrombolysis & & $9(15 \%)$ & & $2(14 \%)$ & 0.999 \\
\hline \multicolumn{6}{|l|}{ Lab resultsafter 24 months } \\
\hline D-dimers $(\mu \mathrm{g} / \mathrm{L})$ & $\mathrm{n}=49$ & $52.0(0.1 ; 283.0)$ & $\mathrm{n}=10$ & $46.2(0.2 ; 1005.0)$ & 0.840 \\
\hline \multicolumn{6}{|l|}{ ECHOafter 24 months } \\
\hline RVIDd (mm) & $\mathrm{n}=59$ & $32.0(22.0 ; 40.0)$ & $\mathrm{n}=14$ & $34.0(29.0 ; 44.0)$ & 0.062 \\
\hline TAPSE (mm) & $\mathrm{n}=59$ & $27.0(19.0 ; 35.0)$ & $\mathrm{n}=14$ & $24.5(15.0 ; 34.0)$ & 0.146 \\
\hline $\mathrm{RAD}(\mathrm{mm})$ & $\mathrm{n}=59$ & $39.0(27.0 ; 52.0)$ & $\mathrm{n}=14$ & $40.5(3.5 ; 47.0)$ & 0.983 \\
\hline TR (degree) & $\mathrm{n}=56$ & $1.0(1.0 ; 2.5)$ & $\mathrm{n}=14$ & $1.0(0.0 ; 2.5)$ & 0.503 \\
\hline PASP estimate $(\mathrm{mmHg})$ & $\mathrm{n}=52$ & $22.5(12.0 ; 44.0)$ & $\mathrm{n}=13$ & $30.0(12.0 ; 55.0)$ & 0.010 \\
\hline \multicolumn{6}{|l|}{ NYHA functional class after 24 months } \\
\hline NYHA 1 & & $39(66.1 \%)$ & & $7(50.0 \%)$ & \\
\hline 2 & & $17(28.8 \%)$ & & $5(35.7 \%)$ & 0.253 \\
\hline 3 & & $3(5.1 \%)$ & & $2(14.3 \%)$ & \\
\hline \multicolumn{6}{|l|}{ Clinical events } \\
\hline Rehospitalization during the study period & & $13(22.0 \%)$ & & $3(21.4 \%)$ & 0.999 \\
\hline Bleeding during the study period & & $5(8.5 \%)$ & & $2(14.3 \%)$ & 0.613 \\
\hline PE recurrence during the study period & & $5(8.5 \%)$ & & $1(7.1 \%)$ & 0.999 \\
\hline At least one clinical event & & $16(27.1 \%)$ & & $5(35.7 \%)$ & 0.527 \\
\hline \multicolumn{6}{|l|}{ Anticoagulation effectiveness } \\
\hline Therapeutic INR values < 75\% & & $2(15.4 \%)$ & & $0(0.0 \%)$ & 0.999 \\
\hline$>75 \%$ & & $11(84.6 \%)$ & & $6(100.0 \%)$ & 0.999 \\
\hline
\end{tabular}

Continuous variables are described using number of patients $(\mathrm{N})$, median $\left(5^{\text {th }}\right.$ and 95 th percentile), differences tested using Mann-Whitney test; Category variables are described using absolute (relative) frequency. Relative frequency is calculated only from available data (excluding patients who were not re-examined). Differences tested using Fisher exact test.

BMI - body mass index. FH VTE - Family history of venous thromboembolism. DVT - deep vein thrombosis. PE - pulmonary embolism. CRP - C-reactive protein. BNP - Brain natriuretic peptide. Hb - hemoglobin. RVIDd - Right ventricle internal diastolic diameter. TAPSE - Tricuspid annular plane systolic excursion. RAD - Right atrium diameter. TR - Tricuspid regurgitation. PG TR - Peak gradient of tricuspid regurgitation. PASP - Peak systolic arterial pressure. INR - international normalized ratio. NYHA - New York Heart Association

defects persisted after 24 months in 14 out of 73 patients $(19.2 \%)$. The high rate of complete reperfusion in this study is in accordance with recent studies ${ }^{9,10,12,14}$, but using the less sensitive CT AG for detection of reperfusion.

One explanation for the relatively high rate of complete reperfusion is the long follow-up period - there was still a decrease in the number of patients with incomplete reperfusion even between months 12 and 24. Another possible explanation may lie in the duration of anticoagulative treatment in our study - most of our patients were treated with anticoagulants for more than a year and even after 2 years, $36.5 \%$ of patients were receiving anticoagulative treatment. The relatively small extent of the initial pulmonary embolisms in our patient group with an average of 
only 3.1 defects per patient could be another explanation. Also, the used methodology could have led to a minor bias - patients in whom perfusion scintigraphy revealed a normal perfusion and who did not have any clinical PE recurrence, did not undergo another scan. It is therefore possible that the occurrence of perfusion defects might have been slightly underestimated when compared with methods used in other papers as some patients might have developed subclinical PE relapse and therefore new perfusion defects. Development of new perfusion defects over time was described e.g. by Ribeiro in $25 \%$ of patients ${ }^{2}$, increase in the number of thrombi detected during subsequent CTAG examinations in $2 \%$ of patients was reported by Alonso-Martinez ${ }^{7}$. In other studies, however, no new perfusion defects were reported and no formation of new perfusion defects was revealed in our patients, either (with the exception of patients with reported clinical recurrence of PE).

The main risk factors of incomplete reperfusion include greater PE extent expressed as obstruction index (odds ratio, OR, 1.34-1.94) (ref..$^{6-9}$ ) or presence of central thrombi during initial CT scan ${ }^{10}$. Various studies highlight mostly the following clinical risk factors: Personal history of cardiovascular disease ${ }^{9}$ or of thromboembolic disease (OR 2.06 - 2.6) (ref. ${ }^{8,9}$ ), increased time from the onset of symptoms to the diagnosis (OR 1.17) (ref. ${ }^{8}$ ), greater age $^{5,7}$, higher degree of PE severity ${ }^{7,12}$, spontaneous PE (OR 1.4) $\left(\right.$ ref. $^{5}$ ), or higher TFPI or factor VIII levels ${ }^{8}$. Studies are however not in mutual agreement on the individual parameters constituting the risk factors of reperfusion disorders. In our study, older age, more sever PE and, interestingly, higher hemoglobin levels were found to be significant predictors of incomplete reperfusion while neither personal history of TED nor spontaneity of PE were confirmed as risk factors.

The reason for the increased rate of reperfusion disorders in older patients has not been studied yet. The likely explanation may lie in the fact that ageing leads to degenerative involutional changes of the vessel walls and of the endothelium in the pulmonary bloodstream, resulting in lower production of vasodilating agents such as prostaglandins and nitrous oxide. Greater PE severity (in our patient group, intermediate or high risk PE) was proved to be a significant risk factor in several studies ${ }^{7,12}$ and is related to the fact that clinically more serious PEs are associated with a greater degree of obstruction of the pulmonary bloodstream, which is in itself a well-known predictor of perfusion disorders ${ }^{6,9}$.

In our study, interestingly, we found that increased hemoglobin levels are another significant risk factor of incomplete reperfusion. As far as we know, this finding has not been reported and has not been object of any other study dealing with pulmonary reperfusion. It is however a well known fact that myeloproliferative diseases including polycythemia are predictors of both arterial and venous thrombosis as well as of CTEPH development following a PE episode ${ }^{15,16}$. The reason for thrombophilia in polycythemia patients is a greater blood viscosity leading to a greater arterial wall shear stress combined with formation of aggregates of red blood cells in arterioles and capil- laries, which in turn leads to activation of platelets, prothrombogenic and pro-inflammatory state and, therefore, to disorders in resolution of thrombi ${ }^{16}$. Neither of these factors are however specific for primary polycythemia and can be associated with any increase in haemoglobin levels, which could be the explanation of our finding.

Persisting reperfusion defects after PE should be related to their clinical significance. The presence of persistent perfusion defects shows only low correlation with echocardiographic signs of elevated pulmonary pressure, with persistent dilatation and dysfunction of the right ventricle, or with reduced functional capacity of patients (rated using NYHA class or 6 minute walk test) (ref. ${ }^{6,8,9}$ ). Only 3 published studies have examined the effect of persistent perfusion disorders in relation to clinical endpoints ${ }^{5,9,10}$. Of these, only Pesavento reported that the persistence of perfusion defects after 3 years of initial TED correlates with combined endpoint of CTEPH development + TED recurrence ${ }^{5}$. No remaining studies have shown any statistically significant correlation of persistent defects with higher incidence of recurrence of pulmonary embolism ${ }^{9}$ or unfavourable patient outlook ${ }^{10}$.

Similarly, our study, despite revealing a correlation of persisting perfusion defects with higher systolic pressure in the pulmonary artery, did not find any correlation of these defects with enlarged right heart compartments, with decreased right ventricular function or with functional abilities evaluated as NYHA class. Similarly, no statistically significant difference in the clinical endpoints such as the number of rehospitalizations or PE recurrences was found between patients with/without reperfusion defects; it is however necessary to note that we did not expect to reveal any such differences due to the size of our patient group.

The principal limitation of the study was small patient sample size precluding evaluation of relations between reperfusion defects and clinical endpoints such as mortality, serious cardiovascular events, TED recurrence and CTEPH. Another limitation could the method of scintigraphy follow-ups, which is discussed above in detail. The use of various anticoagulants may also be perceived as a limitation, albeit a minor one as this study was not focused on anticoagulant evaluation and the size of the study group would not allow any reasonable comparison anyway. The last limitation we can see might be a lacking objective evaluation of the functional capacity of the patients, e.g. using 6 minutes walk test.

\section{CONCLUSION}

During long-term monitoring of patients after an acute episode of pulmonary embolism, persisting perfusion defects were observed in $20(26.0 \%)$ patients after 6 months, $19(25.3 \%)$ patients after 12 months and in 14 (19.2\%) patients after 24 months. This was more frequent in older patients, in patients with higher levels of haemoglobin, higher risk of initial PE (intermediate and high risk) in patients with a greater dimension of the right ventricle and with significant tricuspid insufficiency during the 
initial echocardiographic examination. In patients with perfusion defects persisting at 24 months, a higher systolic pressure in the pulmonary artery was recorded; however, neither larger dimensions of right hand compartments, nor poorer function of the right ventricle in the 24 months echocardiography were detected. No significant difference was found between groups with and without incomplete reperfusion apropos rehospitalization, PE recurrence or functional fitness rated as NYHA class. In 3 (3.5\%) patients, signs of chronic thromboembolic pulmonary hypertension were present after 24 months.

\section{ABBREVIATION}

BMI, Body mass index; BNP, Brain natriuretic peptide; CRP, C-reactive protein; CT, Computed tomography; CT AG, Computed tomography angiography; CTEPH, chronic thromboembolic pulmonary hypertension; DVT, Deep vein thrombosis; ESC, European Society of Cardiology; ECHO, Echocardiography; FH VTE, Family history of venous thromboembolism; Hb, Hemoglobin; INR, International normalized ratio; NYHA, New York Heart Association; PASP, Peak systolic arterial pressure; PE, Pulmonary embolism; PG TR, Peak gradient of tricuspid regurgitation; RAD, Right atrium diameter; RVIDd, Right ventricle internal diastolic diameter; TAPSE, Tricuspid annular plane systolic excursion; TED, Thromboembolic disease; TFPI, Tissue factor pathway inhibitor; TR, Tricuspid regurgitation; VP, ventilationperfusion (scintigraphy).

Acknowledgement: Many thanks to Michal Svoboda for statistical analysis and Vladimir Janovsky and Pavol Bindas for data collection.

Author contributions: JM: study design, data interpretation, manuscript writing and literature search; PJ: study design, data interpretation; JM, JP, JV, LK: data collection.

Conflict of interest statement: The authors declare there are no conflicts of interest regarding the publication of this article.

\section{REFERENCES}

1. Goldhaber SZ, Visani L, De Rosa M. Acute Pulmonary Embolism: Clinical Outcomes in the International Cooperative Pulmonary Embolism Registry (Icoper). Lancet 1999; 353(9162):1386-9.

2. Ribeiro A, Lindmarker $P$, Johnsson $H$, Juhlin-Dannfelt $A$, Jorfeldt $L$.
Pulmonary Embolism: One-Year Follow-up with Echocardiography Doppler and Five-Year Survival Analysis. Circulation 1999;99(10):1325-30.

3. Dentali F, Donadini M, Gianni M, Bertolini A, Squizzato A, Venco A, Ageno W. Incidence of Chronic Pulmonary Hypertension in Patients with Previous Pulmonary Embolism. Thromb Res 2009;124(3):256-8.

4. Pengo V, Lensing AW, Prins MH, Marchiori A, Davidson BL, Tiozzo F, Albanese P, Biasiolo A, Pegoraro C, Iliceto S, Prandoni P. Incidence of Chronic Thromboembolic Pulmonary Hypertension after Pulmonary Embolism. N Engl J Med 2004;350(22):2257-64.

5. Pesavento R, Filippi L, Palla A, Visona A, Bova C, Marzolo M, Porro F, Villalta S, Ciammaichella M, Bucherini E, Nante G, Battistelli S, Muiesan ML, Beltramello G, Prisco D, Casazza F, Ageno W, Palareti G, Quintavalla R, Monti S, Mumoli N, Zanatta N, Cappelli R, Cattaneo M, Moretti V, Cora F, Bazzan M, Ghirarduzzi A, Frigo AC, Miniati M, Prandoni P. Impact of Residual Pulmonary Obstruction on the LongTerm Outcome of Patients with Pulmonary Embolism. Eur Resp J 2017;49(5):1601980. doi: 10.1183/13993003.01980-2016

6. Ribeiro A, Lindmarker $P$, Johnsson $H$, Juhlin-Dannfelt A, Jorfeldt L. Pulmonary Embolism: A Follow-up Study of the Relation between the Degree of Right Ventricle Overload and the Extent of Perfusion Defects. J Intern Med 1999;245(6):601-10.

7. Alonso-Martinez JL, Anniccherico-Sanchez FJ, Urbieta-Echezarreta MA, Garcia-Sanchotena JL, Herrero HG. Residual Pulmonary Thromboemboli after Acute Pulmonary Embolism. Eur J Intern Med 2012;23(4):379-83.

8. Sanchez O, Helley D; Couchon S, Roux A, Delaval A, Trinquart L, Collignon MA; Fischer AM, Meyer G. Perfusion Defects after Pulmonary Embolism: Risk Factors and Clinical Significance. J Thromb Haemost 2010;8(6):1248-55.

9. den Exter PL, van Es J, Kroft LJ, Erkens PM, Douma RA, Mos IC, Jonkers G, Hovens MM, Durian MF, ten Cate $H$, Beenen LF, Kamphuisen PW, Huisman MV. Thromboembolic Resolution Assessed by Ct Pulmonary Angiography after Treatment for Acute Pulmonary Embolism. Thromb Haemost 2015;114(1):26-34.

10. Choi KJ, Cha SI, Shin KM, Lim JK, Yoo SS, Lee J, Lee SY, Kim CH, Park JY, Lee WK. Factors Determining Clot Resolution in Patients with Acute Pulmonary Embolism. Blood Coagul Fibrinolysis 2016;27(3):294-300.

11. Van Rossum AB; Pattynama PM, Tjin ATE, Kieft GJ. Spiral Ct Appearance of Resolving Clots at 6 Week Follow-up after Acute Pulmonary Embolism. J Comp Assist Tomogr 1998;22(3):413-7.

12. Cosmi B, Nijkeuter M, Valentino M, Huisman MV, Barozzi L, Palareti G. Residual Emboli on Lung Perfusion Scan or Multidetector Computed Tomography after a First Episode of Acute Pulmonary Embolism. Intern Emerg Med 2011;6(6):521-8.

13. Meysman M, Everaert H, Buls N, Nieboer K, de Mey J. Comparison of Ventilation-Perfusion Single-Photon Emission Computed Tomography (V/Q Spect) Versus Dual-Energy Ct Perfusion and Angiography (Dect) after 6 Months of Pulmonary Embolism (Pe) Treatment. Eur J Rad 2015;84(9):1816-9.

14. Pesavento R, Filippi L, Pagnan A, Visona A, Pauletto P, Vescovo G, Cuppini S, Beltramello G, Villalta S, De Conti G, Vedovetto V, Frigo AC, Prandoni P. Unexpectedly High Recanalization Rate in Patients with Pulmonary Embolism Treated with Anticoagulants Alone. Am J Resp Crit Care Med 2014;189(10):1277-9.

15. Bonderman D, Jakowitsch J, Adlbrecht C, Schemper M, Kyrle PA, Schonauer V, Exner M, Klepetko W, KneussI MP, Maurer G, Lang I. Medical Conditions Increasing the Risk of Chronic Thromboembolic Pulmonary Hypertension. Thromb Haemost 2005;93(3):512-6.

16. Adir Y, Humbert M. Pulmonary Hypertension in Patients with Chronic Myeloproliferative Disorders. Eur Resp J 2010;35(6):1396-406. 